EPJ manuscript No.

(will be inserted by the editor)

\title{
On the Parisi-Toulouse hypothesis for the spin glass phase in mean-field theory
}

\author{
A. Crisanti ${ }^{1}$ a, T. Rizzo ${ }^{2}$ b, and T. Temesvari ${ }^{3}$ c \\ ${ }^{1}$ Dipartimento di Fisica, Università di Roma La Sapienza, Istituto Nazionale Fisica della Materia Unità di Roma I and SMC, \\ P.le Aldo Moro 2, I-00185 Roma, Italy. \\ 2 SMC-INFM, Dipartimento di Fisica, Università di Roma La Sapienza, P.le Aldo Moro 2, I-00185 Roma, Italy. \\ ${ }^{3}$ HAS Research Group for Theoretical Physics, Eötvös University, Pázmány Péter sétány 1/A, H-1117 Budapest, Hungary.
}

Received: date / Revised version: date

\begin{abstract}
We consider the spin-glass phase of the Sherrington-Kirkpatrick model in the presence of a magnetic field. The series expansion of the Parisi function $q(x)$ is computed at high orders in powers of $\tau=T_{c}-T$ and $H$. We find that none of the Parisi-Toulouse scaling hypotheses on the $q(x)$ behavior strictly holds, although some of them are violated only at high orders. The series is resummed yielding results in the whole spin-glass phase which are compared with those from a numerical evaluation of the $q(x)$. At the high order considered, the transition turns out to be third order on the Almeida-Thouless line, a result which is confirmed rigorously computing the expansion of the solution near the line at finite $\tau$. The transition becomes smoother for infinitesimally small field while it is third order at strictly zero field.
\end{abstract}

PACS. 75.10.Nr Spin-glass and other random models - 02.30.Mv Approximations and expansions

Mean-field theory is commonly used as a starting point tems like the ferromagnet. On the contrary, the glassy (i.e. zeroth order approximation) in the perturbative treat- phase of disordered systems is highly non-trivial even in ment of finite dimensional, short-ranged systems. As such, mean-field theory: in the Ising spin glass, the prototype it is usually very simple, almost trivial, in ordinary sys- of such systems, the thermodynamics of the low tempera-

\footnotetext{
a andrea.crisanti@phys.uniroma1.it

b tommaso.rizzo@inwind.it

c temtam@helios.elte.hu
} ture phase is built on an ultrametrically structured order parameter matrix proposed by Parisi (see [1] and some of 
A. Crisanti et al.: On the Parisi-Toulouse hypothesis for the spin glass phase in mean-field theory

the reprints from this book). Although the region close to the zero magnetic field critical point is easily accessible, to get quantitative results for physical observables deep inside the glassy phase is a rather difficult task.

The survival of the mean-field picture, at least qualitatively, in finite dimensional, short-ranged systems has been a controversial and long debated problem in the last decades. It is clear that a deeper, and more precise, understanding of the mean-field thermodynamics of the spin glass phase is a prerequisite to make any comparison, numerical or analytical, with physical systems. The Parisi-Toulouse (PaT) hypotheses of Ref. 4] (also known as projection hypotheses) were not only a first step in this direction, but had the advantage of simplicity, a common feature of mean-field theory. An extension to systems with an average ferromagnetic interaction followed soon [5], whereas a scaling assumption for the order parameter function $q(x)$ was put forward in [6], see eqs. 6] It was first pointed out in ref. [6] that the whole set of hypotheses cannot be exact, as it leads to inconsistency. An argument was presented in ref. 7] that the projection hypothesis for the magnetization contradicts the maximization rule for the free energy, at least near the instability, AlmeidaThouless (AT), line 8]. An analysis of the exact, diffusionlike differential equations describing the thermodynamics of the mean-field glassy phase was presented in 9. Using series expansions, with relatively short series, it was concluded in this paper that the order parameter function $q(x)$ and the breakpoint $x_{1}$ depend only on temperature, and, among the set of statements of the PaT hypotheses, these are the only ones which may be exact. The order of the transition when crossing the AT-line can also be deduced from the projection hypothesis, and it turns out to be second order in the Ehrenfest sense, at least in finite field 4]. (The magnetic susceptibility has, for example, a finite, albeit small jump.) Nevertheless, subsequent calculations and arguments [7,10] seemed to provide evidence that the transition is third order, just as in zero magnetic field. Recent numerical simulations on the SK model in a field are discussed in [14.

In this work we study the Parisi solution of the SherringtonKirkpatrick (SK) model in the presence of an external field through the methods developed in 12 . In particular, the computation of the high order expansion of $q(x)$ in power of the reduced temperature and of the field allows us to finally answer the problem of the validity of the PaT hypotheses 4,5,6]. The PaT hypotheses are a set of scaling relations concerning the behavior of the function $q(x)$ under changes of the external fields. As we shall briefly recall below, they allow to compute all the quantities of interest of the spin glass phase without solving the Parisi equations but simply using the SK replica symmetric solution evaluated at the AT line [8]. It turned out from our analysis that no one of the PaT statements strictly holds, some of them, however, are so highly consistent with the numerical estimates of the $q(x)$ that their approximate nature can be detected only at high order in the series expansion.

If one evaluates numerically the $q(x)$, one finds that the magnetization seems to be independent of the temperature in the spin glass phase; similarly the self-overlap 
$q_{E A}$ seems to be independent of the magnetic field. This motivated the PaT hypotheses which assume this fact to be exactly true, i.e.

$$
M(H, T)=M(H), \quad q_{E A}(T, H)=q_{E A}(T) .
$$

As a consequence, the free energy is additive, i.e. we have $F(T, H)=F_{1}(T)+F_{2}(H)$. Furthermore, the energy is also additive, while the entropy depends only on the temperature. The previous equations show that if we want to compute $M(H)$, we can compute the magnetization at any temperature in the spin glass phase provided the field is equal to $H$; in particular, it can be computed at the boundary of the spin glass phase, i.e. on the AT line, where the replica symmetric solution is the correct one:

$$
M(H)=M(H, T)=M_{S K}\left(H, T_{A T}(H)\right)
$$

Here the function $H_{A T}(T)$, or its inverse $T_{A T}(H)$, parameterizes the AT line. The argument can be generalized to any quantity that depends on either the temperature, or the magnetic field alone. For instance, we have

$$
\begin{gathered}
S(T)=S(T, H)=S_{S K}\left(T, H_{A T}(T)\right), \\
q_{E A}(T)=q_{E A}(T, H)=q_{S K}\left(T, H_{A T}(T)\right) .
\end{gathered}
$$

Furthermore, if we consider the expression for the energy

$$
E=-\frac{1}{2 T}\left(1-\int_{0}^{1} q^{2}(x) d x\right)-M H
$$

we see that a possible way to have an additive character is to guess proper scaling laws also for $q_{0}(H, T)$ and $q(x, H, T)$. This is at the origin of the full PaT scaling laws:

$$
\left\{\begin{array}{l}
q_{0}(H, T)=q_{0}(H)=q_{S K}\left(H, T_{A T}(H)\right) \\
q(x, T, H)=q(x, T)=q_{u}(x / T) \\
q_{E A}(T, H)=q_{E A}(T)
\end{array}\right.
$$

The universal temperature independent function $q_{u}(y)$ can be computed from the relation $\int_{0}^{1} q(x) d x=1-T$, which holds in zero magnetic field, and the knowledge of $q_{E A}(T)$. Therefore any quantity of interest can be determined simply projecting it from the AT line, where the SK solution is correct, down into the replica symmetry broken (RSB) phase; for this reason the PaT hypotheses are also known as "projection hypotheses". In [6] it was already noticed that the PaT hypotheses must be wrong somehow, this was deduced indirectly comparing the values of different expressions for the specific heat. Instead, here we are able to check the relations (6) directly.

The equations determining $q(x)$ follows from Parisi differential equation [2] and the stationarity of the free energy. They have appeared many times in the literature, see ref. $13,11,12$, therefore we choose don't report them here. Besides the function $q(x)$ they involve two auxiliary functions $P(x, y)$ and $m(x, y)$. Introduced as mathematical tools to perform the computation, these functions have also a physical interpretation: the function $m(x, y)$ represents the magnetization of a given site properly averaged (see chap. $\mathrm{V}$ in Ref. [1]) on a cluster of states with mutual overlap $q(x)$ in presence of a frozen effective field $y$, accordingly $m(1, y)=\tanh (\beta y)$; the function $P(x, y)$ represents the probability distribution function over the disorder of the effective field $y$, in particular $P(1, y)$ represents the 
distribution of the cavity field [1]. These functions have

also received a dynamical interpretation in [13.

We have solved the equations by series expansions around $H=0$ and $T=T_{c}=1$. In the following we report the power series of the quantities of interest at lowest orders. To make easier the comparison between order of magnitude we write down the series expansion in terms of the variables $\tau=1-T$ and $p=(3 / 4)^{1 / 3} H^{2 / 3}$. They are intended to be of the same order of magnitude such that the expressions $O(k)$ below corresponds to terms of the form $\tau^{i} p^{k-i}(i=0, \ldots, k)$. Consistently we have $q_{E A}(T, H)=$ $\tau+O(2)$ and $q_{0}(H, T)=p+O(2)$. The computation has been carried on up to 15 th order in the order parameter by methods similar to those described in Ref. [12]. The only fact to take care is that having two variables to expand on, time and memory grow very fast with order, therefore we reduced the problem to a single-variable one considering temperature-dependent values of the field, i.e. we first computed the expansions at 15th order in $\tau$ assum$\operatorname{ing} p=\tau / m$ for $m=2,3 \ldots 16$, and later we reconstructed the $p$-dependence of the various quantities. The series are supposed to be valid for small $\tau$ and $p$; although they are non-convergent they can be resummed through the methods discussed in Ref. [12] yielding precise results in the whole spin-glass phase.

$$
\begin{aligned}
q_{0}(H, T)= & p\left(1+\frac{2 \tau}{3}-\frac{13 \tau^{2}}{9}+\frac{256 \tau^{3}}{81}\right) \\
& +p^{3}\left(-\frac{4}{5}+\frac{32 \tau}{5}\right)-\frac{56 p^{4}}{27}+O(5), \quad \\
q_{E A}(T, H)= & +\tau^{2}-\tau^{3}+\frac{5 \tau^{4}}{2}-\frac{171 \tau^{5}}{10}+\frac{1077 \tau^{6}}{10} \\
& +p^{5}\left(\frac{8}{5}-\frac{208 \tau}{15}\right)-\frac{16 p^{6}}{9}+O(7),
\end{aligned}
$$

$$
\begin{aligned}
q(x, T, H)- & q(x, T, 0)= \\
& p^{5}\left(\frac{32}{5}-\frac{256 \tau}{15}+\frac{3808 \tau^{2}}{45}\right) \\
+ & p^{6}\left(\frac{-64}{9}+\frac{64 \tau}{3}\right)+\frac{2144}{35} p^{7} \\
& +\left(\frac{-132 p^{5}}{5}+\frac{88 p^{6}}{3}+\frac{448 p^{5} \tau}{5}\right) x \\
& +\frac{312 p^{5} x^{2}}{5}+O(8)
\end{aligned}
$$

$$
\begin{aligned}
f(T, H)= & -(1-\tau) \ln 2-\frac{1}{4}-\frac{\tau}{4}-\frac{\tau^{2}}{4}-\frac{\tau^{3}}{12}+\frac{\tau^{4}}{24} \\
& -\frac{\tau^{5}}{120}+\frac{3 \tau^{6}}{20}-\frac{79 \tau^{7}}{140}+\frac{1679 \tau^{8}}{560}-\frac{2 p^{3}}{3} \\
& +\frac{2 p^{5}}{5}-\frac{8 p^{6}}{27}-\frac{11 p^{7}}{35}+\frac{50 p^{8}}{27} \\
& +p^{5}\left(-\frac{4 \tau}{15}+\frac{34 \tau^{2}}{45}-\frac{872 \tau^{3}}{405}\right) \\
& +p^{6}\left(\frac{8 \tau}{27}-\frac{8 \tau^{2}}{9}\right)+\frac{-52 p^{7} \tau}{105}+O(9) .(10)
\end{aligned}
$$

The expansions of energy and magnetization have been computed from the following formulas $13,9,12$

$$
\begin{aligned}
& M=\int P(x, y) m(x, y) d y \\
& E=-\frac{\beta}{2}\left(1-\int_{0}^{1} q^{2}(x) d x\right)-M H .
\end{aligned}
$$

It turned out that they verify all the usual thermodynamic relations such as $S=-\partial f / \partial T^{1}$ and $m=-\partial f / \partial H$.

¿From our analysis the following conclusions can be drawn regarding the PaT scaling hypothesis.

\footnotetext{
${ }^{1}$ In Ref. 9], it was suggested that this thermodynamic relation may not be valid in the spin glass phase. The argument was based on an extrapolation of relatively low order results. It turns out from the analysis of the higher order terms that quantities which were assumed in 9 to depend on the temperature alone, do have a magnetic field dependence too. These findings change the conclusions basically, leading to a support of the validity of that thermodynamic relation.
} 
A. Crisanti et al.: On the Parisi-Toulouse hypothesis for the spin glass phase in mean-field theory

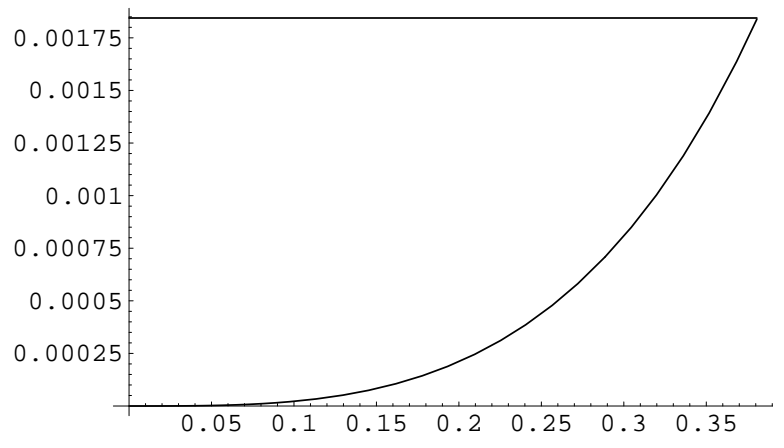

Fig. 1. Plot of $q_{E A}(T, H)-q_{E A}(T, 0)$ at fixed $T=.6$ as a function of $H$ for $0 \leq H \leq H_{A T}(T)=.3826$, the straight line is $q_{S K}\left(T, H_{A T}(T)\right)-q_{E A}(T, 0)=.50689-.50504=.00184$ at $T=$ .6. The data were obtained by the point-by-point procedure for multivariate expansions 12 using the $(7,7)$ Padé approximant. For small $H$, the data fit a power-law behavior .053854 $H^{10 / 3}$.

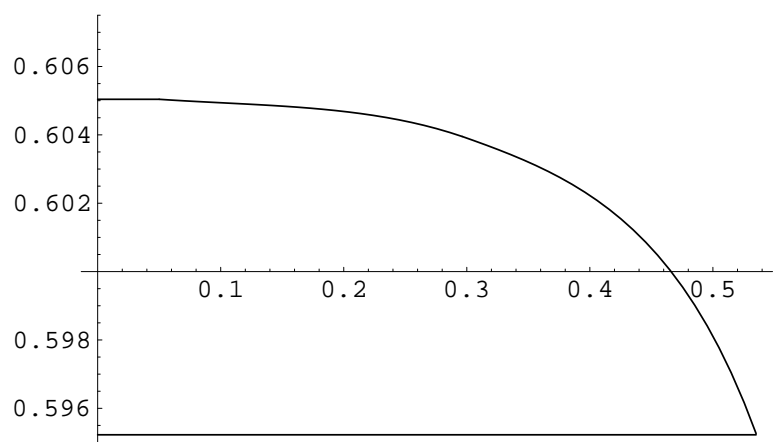

Fig. 2. Plot of $q_{0}(H, T)$ at fixed $H=.5$ as a function of $T$ for $0 \leq T \leq T_{A T}(H)=.5351$, the straight line is $q_{S K}\left(H, T_{A T}(H)\right)=.5951$ at $H=.5$. The data were obtained by the point-by-point procedure for multivariate expansions [12] using the $(7,7),(6,8)$ and $(8,6)$ Padé approximants.

$-q_{E A}(T, H)=q_{E A}(T)$. This scaling law is violated in the expansion in power of $\tau$ and $p$ at order $p^{5}$ (i.e. $\left.H^{10 / 3}\right)$ which means fifth order in the height $q_{0}(T, H)$ of the first plateau. As a consequence, such a violation is hardly seen numerically, and the PaT approxima- tion $q_{E A}(T)=q_{S K}\left(T, H_{A T}(T)\right)$ is excellent at high temperature. We note that if this relation were true, the expansion of $q_{E A}(T)$ around $T=0$ should read $q_{E A}(T)=1-3 / 2 T^{2}+O\left(T^{3}\right)$. This is the expression which is valid on the AT line while the true expression is $q_{E A}(T)=1-\alpha T^{2}+O\left(T^{3}\right)$ with $\alpha=1.60 \pm .01$ obtained resumming the expansion in power of $\tau$ and numerically [12]. In figure 1 we plot $q_{E A}(T, H)-q_{E A}(T, 0)$ at fixed $T$ as a function of the magnetic field. The data were obtained from the series expansions by the resummation procedures discussed in Ref. [12.

$-q(x, T)=q_{u}(\beta x)$ is violated near the critical temperature, as can be seen from the series expansion [12. However, the scaling is a very good approximation in a wide range of temperatures. It becomes exact in the $\operatorname{limit} T \rightarrow 0$ and $x / T \rightarrow 0$. A consequence of this scaling law would be $\lim _{T \rightarrow 0} x_{1}=1 / 2$ [], while the real value turns out to be $.548 \pm .005$ [12. From the above power series it can be also deduced that $x_{1}(T, H)$ picks up a small dependence on $H$, again proportional to $p^{5}$ (i.e. $\left.H^{10 / 3}\right)$.

$-q(x, T, H)=q(x, T)$. This scaling law is violated in the expansion in power of $\tau$ and $p$ at order $p^{5}$ (i.e. $\left.H^{10 / 3}\right)$ which means fifth order in the height $q_{0}(T, H)$ of the first plateau. As for $q_{E A}(T)$, the deviation is very difficult to be seen numerically. Notice that this scaling law is also valid near $T_{c}$ when the more stringent $q(x, T)=q_{u}(x / T)$ is not verified. We have checked at 15th order that all the coefficients in the expansion of $q(x, T, H)$ in power of $x$ pick up corrections propor- 
tional to $p$ such that the lowest power of $p$ is always $p^{5}$ (i.e. $H^{10 / 3}$ ). This is likely to remain true at all orders. $-q_{0}(H, T)=q(H)$. This scaling law is violated too. However, at all values of the magnetic field the behavior of the function $q_{0}(H, T)$ is similar to that of figure 2 obtained by resumming the series expansion. Its value increases linearly from the PaT value at $T=$ $T_{A T}(H)$ while lowering the temperature, but it goes rapidly to a constant value at low temperature. Furthermore, at all fields the difference between the PaT value $q_{0}\left(H, T_{A T}(H)\right)$ and $q_{0}(H, 0)$ is always of order $10^{-2}$. Actually, this is the only violation of the PaT scaling which can be easily seen numerically.

- $F(T, H)=F_{1}(T)+F_{2}(H)$. The first term in the expansion in power of $\tau$ and $p$ which violates this additive law is $\tau p^{5}$ i.e. $\tau H^{10 / 3}$, a sixth order term. As a consequence, the deviation from the PaT entropy (3) is only fifth order, and again it is a very good approximation. The same is true for the PaT magnetization (2). We have checked at very high order in the expansion that the lowest power of $p$ in the mixed terms is always $p^{5}$ (i.e. $H^{10 / 3}$ ), i.e. fifth order in the height $q_{0}$ of the first plateau, a result that is likely to be valid at all temperatures, explaining the goodness of the PaT approximation in the whole RSB phase. Indeed, it turns out that the approximation $q(x, T, H)=q(x, T, 0)$ breaks down at fifth order in the expansion in powers of $q_{0}$ at a given finite $\tau$. Therefore we expect that at all temperatures the corrections to the PaT estimates are of order $q_{0}^{5}$, and become relevant only at very high fields.
In particular, the field may be expressed up to fourth order in $q_{0}$ in terms of the function $q(x, T, 0)$, we have indeed $h^{2}=2 /\left(3 T q^{\prime}(0, T)\right) q_{0}^{3}+0 q_{0}^{4}+O\left(q_{0}^{5}\right)$; this explains why the scaling $q_{0}(H, T)=q(H)$ is best verified at low temperature, since $T q^{\prime}(0, T)$ tends to the constant value $0.743 \pm 0.002$ for $T \rightarrow 0$ [12]

The predictions from the perturbative expansions have been compared with those from the numerical solution of the equations for the $q(x)$ using the high precision technique introduced in ref. 12 . By comparing the $H=0$ with the $H \neq 0$ solution in all cases we found a rather good agreement with analytical results and the violation of the PaT scaling can be appreciated.

The series expansion at high orders allows us to safely determine the order of the transition on the AT line. By making an expansion of the replica symmetric (RS) solution near the transition line the following conclusions can be drawn.

- The RS-RSB transition is third order, i.e. all the possible derivatives of the free energy (temperature, field and mixed) up to second order, inclusively, are continuous along the AT line. All higher order derivatives are discontinuous, and not divergent, at a generic point on the AT line.

- Near the critical point $(\tau=0, H=0)$ the situation is more complicated. First of all, we note that the RS free energy is singular at this point, therefore its derivatives depend on how this point is approached. For instance, temperature derivatives of the RS free energy higher than the fifth are divergent approaching the critical 
point on the AT line, while they are regular on the line $(H=0, T>1)$.

- Approaching the critical point $(\tau=0, H=0)$ along the AT line, the discontinuity in the third field derivative goes to zero as $\sqrt{\tau}$, while the fourth derivative of the RS and RSB free energy (and also their difference) diverge. This fourth derivative with respect to the magnetic field is essentially the nonlinear susceptibility, and it can be expressed, by a fluctuationdissipation-like formula, in term of the so-called longitudinal correlation function $\left(G_{L}\right)$. $G_{L}$ becomes identical with the spin glass susceptibility in exactly zero magnetic field, and diverges at $T_{c}(\tau=0)$, although it remains finite elsewhere.

- The discontinuities in the third and fourth temperature derivatives also go to zero while approaching the critical point along the AT line, only the fifth temperature derivative has a finite discontinuity in this limit. As already mentioned, higher temperature derivatives of the RS free energy diverge at $T_{c}$, while the RSB derivatives are regular. Therefore, if we consider only temperature derivatives, the RS-RSB transition becomes fifth order near the critical point. This can be understood noticing that, while approaching the critical point from the left along the AT line, the RS solution, valid above the line, is similar to the (unstable) RS solution at $T<1$ and $H=0$ whose free energy differs from the RSB free energy at fifth order in $\tau$. This behaviour of the temperature derivatives on the AT line in the $H \rightarrow 0$ limit is markedly different from that at $H$ strictly zero. Indeed at $H=0$ we must consider the $T>1 \mathrm{RS}$ solution whose free energy differs from the RSB free energy at third order in $\tau$, as can be checked using $F_{R S}=-1 /(4 T)-T \log 2$ valid for $T>1$

These results were obtained analyzing very long series and are rather safe, however to confirm them we performed a series expansion of the RSB solution near the AT line in power of $d h=h-h_{A T}$ at fixed temperature. This is an improvement on the result of [10] where such an expansion was obtained using a 1 RSB function instead of the correct full RSB $q(x)$. This expansion allows to compute exactly the derivatives of the RSB free energy on the AT line. As expected we have found that the first and second field derivatives of the RS and RSB free energies are continuous on the AT line. In general we have observed that in order to determine the free energy at order $d h^{n}$ it is sufficient to know $q(x)$ at order $d h^{n-2}$ instead of order $d h^{n-1}$ which would be expected by power counting. Therefore the RS solution, which is the zeroth order approximation, gives the correct free energy up to second order in $d h$. The coefficients of this expansions depend on integrals of the form

$$
\overline{m^{j}}=\frac{1}{\sqrt{2 \pi q}} \int_{-\infty}^{+\infty} e^{-y^{2} /(2 q)} \tanh [\beta y+\beta H]^{j} d y
$$

evaluated on the AT line. For instance the second field derivative reads

$$
\begin{aligned}
\frac{\partial^{2} F}{\partial H^{2}}(T & \left., H_{A T}(T)\right)=\frac{\beta}{1+\left(q_{A T}-1\right) \beta^{2}} \\
& \times\left(q_{A T}-1-2 q_{A T} \beta^{2}+q_{A T}^{2} \beta^{2}\right. \\
& \left.-\beta^{2}\left(-1+M_{A T}^{2}-2 M_{A T} \overline{m^{3}}+{\overline{m^{3}}}^{2}\right)\right)
\end{aligned}
$$


The breakpoint reads

$$
x_{1}\left(T, H_{A T}(T)\right)=\frac{2\left(-2+2 \beta^{2}-3 q_{A T} \beta^{2}+\beta^{2} \overline{m^{6}}\right)}{3-2 \beta^{2}+3 q_{A T} \beta^{2}-\beta^{2} \overline{m^{6}}}
$$

It is interesting to notice that the $T \rightarrow 0$ limit of the breakpoint is exactly $1 / 2$ as predicted by the PaT hypotheses in an independent way and at variance with the $(T=0, H=0)$ value which is .548 $\pm .005[12$.

\section{Acknowledgments}

One of us (T. T.) acknowledges support from the Hungarian Science Fund (OTKA), Grant No. T032424.

\section{References}

1. M. Mézard, G. Parisi, and M.A. Virasoro, "Spin glass theory and beyond", World Scientific (Singapore 1987).

2. G. Parisi, J. Phys. A 13, (1980) L115.

3. G. Parisi, J. Phys. A 13, (1980) 1887.

4. G. Parisi and G. Toulouse, J. Physique Lett. 41, (1980) L361.

5. G. Toulouse, J. Physique Lett. 41, (1980) L447.

6. J. Vannimenus, G. Toulouse and G. Parisi, J. Physique 42, (1981) 565 .

7. G. Toulouse, M. Gabay, T.C. Lubensky and J. Vannimenus, J. Physique Lett. 43, (1982) L109.

8. J. R. L. de Almeida and D. J. Thouless, J. Phys. A 11, (1978) 983.

9. T. Temesvári, J. Phys. A 22, (1989) L1025.

10. H.-J. Sommers, J. Phys. A 17, (1984) 2351.

11. H.-J. Sommers, J. Physique Lett. 46, (1985) L779.

12. A. Crisanti and T. Rizzo, Phys. Rev. E 65, (2002) 046137.
13. H.-J. Sommers, W. Dupont, J. Phys. C 17, (1984) 5785.

14. A. Billoire and B. Coluzzi, Phys. Rev. E 67, (2003) 036108. 